\title{
Expression of kisspeptin and KISS1 receptor in pituitary neuroendocrine tumours - an immunohistochemical study
}

\author{
Milena Mihajlović, Sandra Pekićn, Mirjana Doknić2, Marko Stojanović2, Dragana Miljić2, \\ Ivan Soldatović, Tatjana Vukotić, ${ }^{4}$ Tijana Janev $^{4}$, Sanja Ćirović, ${ }^{5}$ Tatjana Terzićs, Savo Raičević, \\ Milica Skender-Gazibara ${ }^{4}$, Vera Popović, Emilija Manojlović-Gačićs \\ ${ }^{1}$ Department of Pathology, Clinical Centre of Serbia, Belgrade, Serbia \\ ${ }^{2}$ Neuroendocrine Department, Clinic for Endocrinology, Diabetes, and Metabolic Diseases, Clinical Centre of Serbia, \\ Faculty of Medicine, University of Belgrade, Belgrade, Serbia \\ ${ }^{3}$ Institute of Medical Statistics and Informatics, Faculty of Medicine, University of Belgrade, Belgrade, Serbia \\ ${ }^{4}$ Faculty of Medicine, University of Belgrade, Belgrade, Serbia \\ ${ }^{5}$ Institute of Pathology, Faculty of Medicine, University of Belgrade, Belgrade, Serbia \\ ${ }^{6}$ Neurosurgery Clinic, Clinical Centre of Serbia, Belgrade, Serbia
}

\begin{abstract}
Introduction: Pituitary neuroendocrine tumours (PitNETs), traditionally designated as pituitary adenomas, show relatively frequent invasive growth with exceptional metastatic potential, the causes of which are not entirely elucidated. Kisspeptins, which perform their activity through KISS1 receptor (KISS1R), are recognised as metastatic suppressors in many malignant tumours. This study aimed to investigate the immunohistochemical expression of kisspeptin and KISS1R in different types of PitNETs and to compare it with the expression in the normal anterior pituitary, using tissue microarray.

Material and methods: The experimental group consisted of 101 patients with PitNETs, with $45(37.3 \%)$ being of gonadotroph, 40 (33.9\%) somatotroph, $4(3.4 \%)$ corticotroph, 4 (3.4\%) thyrotroph, $3(2.5 \%)$ lactotroph, and $6(5.1 \%)$ null-cell type. The control group consisted of anterior pituitary tissue accidentally removed during the surgery for PitNETs in 17 patients.

Results: Kisspeptin expression was observed in both experimental and control groups, without statistically significant differences in the staining intensity. Negative kisspeptin staining was detected in $10(9.9 \%)$, weak in $79(78.2 \%)$, and moderate in 12 tumours $(11.9 \%)$; none of the tumours had strong staining intensity. The weak staining intensity was predominant in all PitNET types except thyrotroph tumours. Significant statistical difference in terms of kisspeptin expression between types of PitNET and the control group was not observed. Immunohistochemical expression of KISS1R was not observed in the control group or in the experimental group.

Conclusions: We conclude that immunohistochemistry, as a method, cannot confirm the involvement of kisspeptin in tumourigenesis and aggressiveness of PitNETs, but potentially supports its antimetastatic role. The absence of KISS1R immunohistochemical expression in all anterior pituitaries and PitNETs in our cohort needs verification through the use of different procedures designed for the detection of the presence and localisation of proteins in the cell. (Endokrynol Pol 2021; 72 (2): 91-96)
\end{abstract}

Key words: kisspeptin; KISS1 receptor; pituitary adenoma; PitNET; immunohistochemistry

\section{Introduction}

Pituitary neuroendocrine tumours (PitNETs) [1], or traditionally pituitary adenomas, are the most common but heterogenous tumours of the adenohypophysis, with relatively frequent invasive growth and with exceptional metastatic potential [2]. The invasiveness of PitNETs, which leads to the significant morbidity, is a complex and still unclear process, at least partially governed by angiogenesis, degradation of extracellular matrix, epithelial-to-mesenchymal transition, and hypoxia [3], and related to PitNET-type [4]. The essence of aggressive biological behaviour and ma- lignant transformation of PitNETs is still not entirely understood [5].

Kisspeptins are products of the KISS1 gene [6, 7]. They are cleaved into peptides $54,14,13$, and 10 amino acids long, which function through binding to the same receptor [8], named KISS1R (originally named GPR45) [9]. Many studies showed that KISS1 plays the role of a metastasis suppressor gene, loss of which was observed during progression and metastasis in many malignant tumours (including melanoma, gastric, ovarian, endometrial, and bladder carcinoma) [10]. Nevertheless, these conclusions are not unanimous, because the elevated expression of kisspeptins in hepatocellular 
carcinoma was correlated with worse prognosis [11], and the association of kisspeptin expression and progression in breast carcinoma is dependent upon the presence of receptor status [12,13].

Further investigations of kisspeptins and KISS1R in pituitary gland revealed that they also play an important physiological function in the hypothalamic-pituitary-gonadal signalling axis. Being produced by KISS1 neurons of the hypothalamus, kisspeptins activate hypothalamic gonadotropin-releasing hormone (GnRH) neurons through KISS1R, which stimulates production of follicle stimulating hormone (FSH) and luteinising hormone (LH) in the pituitary gonadotropic cells, giving signals for puberty and sexual maturation [14].

Previous studies of kisspeptin and KISS1R performed on the tissue of human PitNETs analysed the presence of their mRNA, using Reverse Transcriptase Polymerase Chain Reaction (RT-PCR). KISS1R transcripts were observed in the normal pituitary and PitNETs in both investigations $[15,16]$. Results regarding kisspeptin were contradictory; kisspeptin transcripts were detected in both anterior pituitary and PitNETs in one investigation [15], whereas they were absent in the other [16]. To the best of our knowledge, the presence of kisspeptin and KISS1R in human anterior pituitary and PitNETs has not yet been explored by immunohistochemistry (IHC).

The aim of this study was to explore the immunohistochemical expression of kisspeptin and KISS1R in tissue of different types of PitNETs and to compare it with the expression in the normal anterior pituitary.

\section{Material and methods}

The samples of PitNETs were provided from patients treated neurosurgically at the Neurosurgery Clinic, Clinical Centre of Serbia, Belgrade.

Tumours were classified according to WHO classification [4], regarding IHC expression of anterior pituitary hormones and transcription factors, steroidogenic factor-1 (SF1), and pituitary-specific transcription factor 1 (Pit-1). The antibody for the transcriptional factor T-Pit (T-box family member TBX19) was unavailable.

The experimental group consisted of 101 patients, 54 of whom were male $(53.5 \%)$ and 47 female $(46.5 \%)$. The age of patients at the moment of surgery ranged between 20 and 80 years (mean $53 \pm 13.9$ ). Forty-four patients $(43.6 \%)$ had gonadotroph tumour, 40 patients $(39.6 \%)$ had somatotroph tumour, $6(5.9 \%)$ had null cell tumour, 4 (4\%) had corticotroph tumour, 4 (4\%) had thyrotroph tumour, and $3(2.9 \%)$ had lactotroph tumour.

The control group consisted of tissue of the anterior pituitary accidentally removed during the surgery for PitNETs in 17 patients (4 males [23.5\%] and 13 females [76.5\%]) with the age ranging between 34 and 70 years (mean $47.6 \pm 12.3$ ). The experimental and control groups were matched by age $(p=0.095)$.

All the experiments were reviewed and approved by the local Ethics Committee of the Medical Faculty, University of Belgrade, Belgrade, Serbia and were in accordance with the Declaration of Helsinki.

\section{Preparation of the tissue and tissue microarray (TMA)}

The fixation of the samples of both the experimental and control groups was performed in buffered $10 \%$ formalin, dehydrated in graded ethanol, and submerged in paraffin blocks. Three representative areas of each sample were recognised on haematoxylin-eosinstained slides and selected for TMA construction with 1.2-mm cores [17]. The presence of at least one core was regarded as sufficient for the analysis.

\section{Immunohistochemistry}

As advised by the manufacturer, the IHC was done on the 5- $\mu \mathrm{m}$ sections of TMA with the following antibodies: anti-cytokeratin 8 (CK8) (Leica Biosystems, clone TS1, 1:50), anti-growth hormone (GH) (DAKO, polyclonal, 1:400), anti-prolactin (PRL) (DAKO, polyclonal, 1:300), anti-FSH (Immunotech, polyclonal, 1:3000), anti-LH (DAKO, clone C93, 1:50), anti-adrenocorticotropic hormone (ACTH) (DAKO, clone 02A3,1:100), SF-1 (DAKO, Invitrogen, clone N1665, 1:200), Pit-1 (DAKO, Novus Biologicals, polyclonal, 1:500), anti-kisspeptin antibody (ab72804 polyclonal, 1:100), and Anti-KiSS1 receptor antibody (ab140839 polyclonal, $14 \mathrm{mg} / \mathrm{mL}$ ). Appropriate positive controls were used in order to standardise all immunostains (tissue of anterior pituitary for the pituitary hormones, SF-1, Pit-1, and CK8 and placental tissue for kisspeptin and KiSS1R). Immunohistochemistry for SF-1 and Pit-1 was performed using DAKO autostainer. For the remaining antibodies, IHC was performed manually, using an Ultravision Detection System, Large Volume, Anti-Polyvalent HRP (Thermo Scientific). Chromogen for all the stains was 3,3'-diaminobenzidine. Negative control was achieved omitting the primary antibody. Staining on the same run was performed for all antibodies to avoid inter-assay variability.

\section{Quantification of the IHC Stains}

Immunohistochemistry stain was taken into account as cytoplasmic for kisspeptin and membranous for KISS1R. Two pathologists (EMG and MM) established the definition of the kisspeptin staining intensity and chose a few representative cases of negative, weak, moderate, and strong intensity (Fig. 1).

Staining intensity for KISS1R was not established due to the negative staining in all cases in the experimental and control groups.

\section{Statistical analysis}

For the statistical analysis, for comparison of differences between the groups, Mann-Whitney U and Mantel-Haenszel chi-square tests were performed. $\mathrm{P}$ values less than 0.05 were considered significant. All the data were analysed by SPSS 20.0 (IBM corp.) statistical software.

\section{Results}

Cytoplasmic expression of kisspeptin was registered in both the experimental and the control group (Fig. 1). In the experimental group, staining was negative in 10 tumours $(9.9 \%)$, weak in $79(78.2 \%)$ tumours, and moderate in 12 tumours $(11.9 \%)$; strong staining intensity was not observed. In the control group, staining was weak in $14(82.4 \%)$ and moderate in $3(17.6 \%)$ samples; negative staining was not observed. Statistically significant differences between the experimental and the control group, in terms of kisspeptin expression, were not observed ( $\mathrm{p}=0.199)$.

Regarding the expression of kisspeptin in different types of PitNETs, weak staining intensity was predomi- 
Table 1. Kisspeptin immunohistochemical expression in the anterior pituitary (control group) and types of pituitary neuroendocrine tumours (PitNETs), and the results of the comparison of the kisspeptin staining intensity between anterior pituitary (control group) and types of PitNETs

\begin{tabular}{|c|c|c|c|c|}
\hline \multirow{2}{*}{ Groups: } & \multicolumn{3}{|c|}{ Kisspeptin expression } & \multirow{2}{*}{$\begin{array}{c}\text { p value } \\
\text { (control vs. experimental) }\end{array}$} \\
\hline & Negative & Weak & Moderate & \\
\hline \multicolumn{5}{|l|}{ Control } \\
\hline Anterior pituitary & $0(0.0 \%)$ & $14(82.4 \%)$ & $3(17.6 \%)$ & \\
\hline \multicolumn{5}{|l|}{ Experimental } \\
\hline Lactotroph & $1(33.3 \%)$ & $2(66.7 \%)$ & $0(0.0 \%)$ & 0.148 \\
\hline Gonadotroph & $5(11.4 \%)$ & $36(81.8 \%)$ & $3(6.8 \%)$ & 0.095 \\
\hline Corticotroph & $1(25.0 \%)$ & $3(75.0 \%)$ & $0(0.0 \%)$ & 0.185 \\
\hline Thyrotroph & $0(0.0 \%)$ & $1(25.0 \%)$ & $3(75.0 \%)$ & 0.053 \\
\hline Null-cell & $0(0.0 \%)$ & $4(66.7 \%)$ & $2(33.3 \%)$ & 0.576 \\
\hline Somatotroph — all subtypes & $3(7.5 \%)$ & $33(82.5 \%)$ & $4(10.0 \%)$ & 0.297 \\
\hline Sparsely granulated & $2(11.1 \%)$ & $14(77.8 \%)$ & $2(11.1 \%)$ & \\
\hline Densely granulated & $1(4.5 \%)$ & $19(86.4 \%)$ & $2(9.1 \%)$ & \\
\hline
\end{tabular}

${ }^{*}$ Results are presented as count (\%)

nant in all PitNET types except thyrotroph tumours (Tab. 1, Fig. 1). The comparison between types of PitNET and control group in terms of kisspeptin expression did not reach statistical significance (Tab. 1). Comparison between the different types of PitNETs was not performed due to the small size of some subgroups, which would potentially lead to incorrect results.

Differences between genders regarding kisspeptin IHC expression in PitNETs were not observed $(p=0.212)$.

Immunohistochemical expression of KISS1R was not observed in the control group (anterior pituitary) or in the experimental group, although intensive positive staining was present in the external positive control (placental tissue) (Fig. 1).

\section{Discussion}

Immunohistochemical analysis in this study revealed predominantly weak positivity of kisspeptin and the absence of KISS1R expression in PitNETs, without significant differences in staining compared to the expression in the anterior pituitary (control group).

Although PitNETs are classified as benign neoplasms, they can show aggressive biological behaviour through the invasion of the surrounding structures, which is especially seen in some types (lactotroph PitNETs in males, silent corticotroph PitNETs, sparsely granulated somatotroph PitNETs, plurihormonal Pit-1 positive PitNET, and Crooke cell adenoma) [4]. Incomplete understanding of the causes of the aggressive biological behaviour of basically benign neoplasms, associated with exceptional development of metastases, inspired us to investigate how kisspeptin, widely known to participate in metastasis suppression, plays a role in PitNETs. Predominantly low kisspeptin immunohistochemical expression in PitNETs and anterior pituitary, which was without statistical difference compared to the control group of anterior pituitaries, suggests that the IHC method cannot confirm its role in the process of tumourigenesis of PitNETs. The decrease of kisspeptin expression in tumour tissue, compared to normal tissue, previously observed in the colorectal carcinoma [18], non-small cell lung carcinoma [19], and bladder cancer [20] advocates the involvement of the loss of kisspeptin expression in cancerogenesis. In our study, negative kisspeptin expression was observed only in the minority of tumours, including 1 lactotroph in a male, 5 gonadotroph tumours, 1 corticotroph tumour, and 3 somatotroph tumours (of which 2 were sparsely and 1 was densely granulated) (Tab. 1). Bearing in mind the small proportion of kisspeptin-negative PitNETs in our cohort, of which only some belong to potentially aggressive types [4], and that the aggressiveness of the other kisspeptin-negative PitNET cannot be excluded due to the lack of the clinicopathological correlation in our investigation, we speculate that the IHC method is not able to endorse the involvement of kisspeptins in the aggressiveness of PitNETs. However, the presence of kisspeptin positivity in $90.1 \%$ tumours in our study (although the majority of them were of weak intensity) potentially supports the antimetastatic role of kisspeptin in PitNETs, considering that their metastatic potential is extremely low in spite of the aggressive biological behaviour of some subtypes. Further investigation of the involvement of the kisspeptin/KISS1R system 

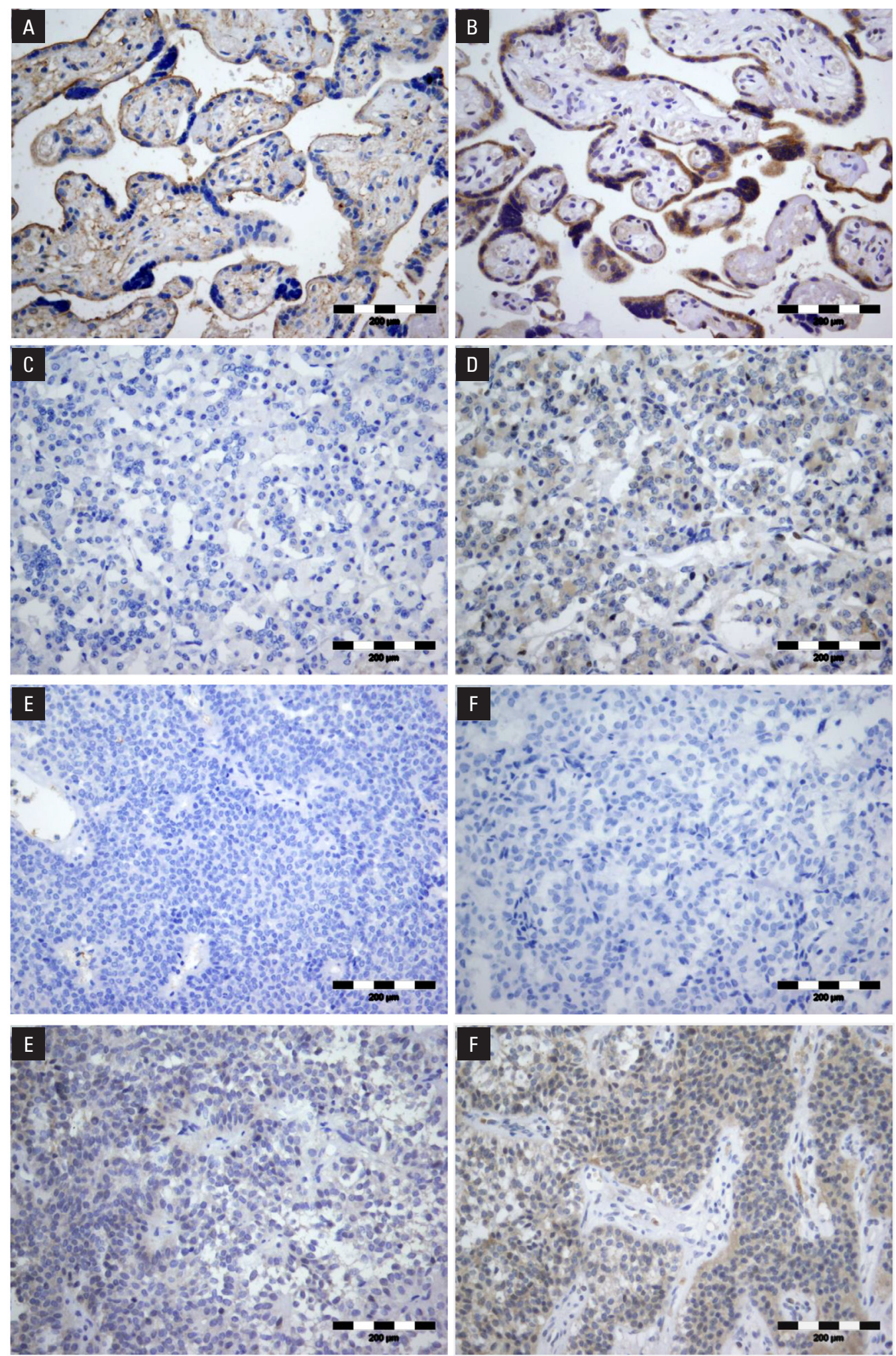

Figure 1. Immunohistochemical expression of kisspeptin and KISS1R in the placental tissue (control), anterior pituitary, and pituitary neuroendocrine tumours (PitNETs). Placental tissue showed intensive membranous positivity for KISS1R (A) and cytoplasmic for kisspeptin (B). The anterior pituitary showed negative staining for KISS1R (C), whilst kisspeptin positivity was predominantly weak (D). PitNETs were immunonegative for KISS1R (E). Kisspeptin immunopositivity in PitNETs was negative (F), weak (G), and moderate (H). All microphotographs were performed with magnification $\times 400$

in PitNET types with clinically confirmed aggressive biological behaviour and pituitary carcinomas, which were not included in this study, would be advised.
The tumour microenvironment and its intrinsic biology are likely to be crucial factors for the influence of kisspeptins and KISS1R on tumour behaviour, 
invasiveness, and metastatic potential [10, 21], which is particularly seen in thyroid tumours, where the aggressiveness and stage of different types of thyroid carcinomas could be related to kisspeptin/KISS1 expression [22, 23]. PitNETs are rather heterogenous types of tumours, classified according to their lineages of differentiation and hormone production [24]. Even though statistical analysis of the potential differences in IHC expression of kisspeptin between PitNET types was not performed in our study (due to the bias risk from small sample numbers in some subgroups, which could lead to incorrect conclusions), predominant weak positivity was observed in all groups. This finding suggests the potential absence of the correlation between the lineage of differentiation and/or hormone production and IHC expression of kisspeptin in PitNETs.

Kisspeptins perform their function presumably through KISS1R [8]. Intriguingly, investigations in cell culture models suggest that the effect of kisspeptins to metastasis suppression is not necessarily driven through KISS1R [25]. Our immunohistochemical findings of the absence of KISS1R in both anterior pituitary and various types of PitNETs are in disagreement with the previous immunohistochemical investigations of KISS1R in anterior pituitary in rats [26] and cell culture of human pituitary adenoma cells [15], discouraging potential treatment of aggressive PitNETs with kisspeptin analogues, previously proven in cell lines and preclinical models of cancers [10]. Nevertheless, it is known that of the many commercial antibodies for IHC only a few have gained the trust of pathologists and are used in routine practice. Even though the polyclonal antibody against KISS1R used in our investigation gave negative results in experimental tissue with positive control in placental tissue, it would be beneficial to perform additional immunohistochemical studies with multiple types of IHC antibodies against KISS1R, to verify the presence of KISS1R on the cell membrane of PitNETs.

The investigation of the presence and the function of any protein is very complex, requiring the use and validation by multiple detection systems. The discrepancy in the expression of KISS1R in PitNETs and anterior pituitary between our investigation, where it was not detected by IHC, and previous investigations, where KISS1R mRNA was detected by RT-PCR [15,16], could be explained by differences in methodological approach. Namely, the amount of mRNA in a cell is not in direct proportion to the amount of its transcribed protein in a cell, because some mRNAs remain untranslated and some are translated suboptimally. Furthermore, translation rates are also diverse among different mRNA species [27]. On the other hand, IHC is a highly sensitive and specific method for the detection of proteins in the tissue, whose results could be obscured by preanalyti- cal conditions, such as the quality of tissue fixation, or mis-interpreted, because read-outs of the results of IHC are prone to subjective interpretation [28, 29]. Nevertheless, disagreement in kisspeptin expression in PitNETs and anterior pituitary in our IHC study with one [15], but not with the other [16], RT-PCR study emphasises the need for further investigation, in which simultaneous detection of mRNA (by RT-PCR) and its transcript protein (by IHC) would be performed.

We conclude that immunohistochemistry, as a method, cannot confirm the involvement of kisspeptin in the tumourigenesis and aggressiveness of PitNETs, but potentially supports its antimetastatic role. The absence of KISS1R immunohistochemical positivity in all anterior pituitaries and PitNETs in our cohort needs verification through the use of different types of antibodies and procedures designed for the detection of the presence and localisation of proteins in the cell.

\section{Acknowledgements}

This work was supported by the Ministry of Education and Science of Republic of Serbia, Grant No. 175033.

\section{References}

1. Asa SL, Casar-Borota O, Chanson P, et al. attendees of 14th Meeting of the International Pituitary Pathology Club, Annecy, France, November 2016. From pituitary adenoma to pituitary neuroendocrine tumor (PitNET): an International Pituitary Pathology Club proposal. Endocr Relat Cancer. 2017; 24(4): C5-C8, doi: 10.1530/ERC-17-0004, indexed in Pubmed: 28264912.

2. Raverot G, Burman P, McCormack A, et al. European Society of Endocrinology. European Society of Endocrinology Clinical Practice Guidelines for the management of aggressive pituitary tumours and carcinomas. Eur J Endocrinol. 2018; 178(1): G1-G24, doi: 10.1530/EJE-17-0796, indexed in Pubmed: 29046323.

3. Yang Qi, Li X, Yang Qi, et al. Molecular Network Basis of Invasive Pituitary Adenoma: A Review. Front Endocrinol (Lausanne). 2019; 10: 7, doi: 10.3389/fendo.2019.00007, indexed in Pubmed: 30733705.

4. Lloyd RV, Osamura RY, Kloppel G, Rosai J. World Health Organization classification of tumours of endocrine organs. 4th ed. IARC Press, Lyon 2017.

5. Trouillas J, Burman P, McCormack A, et al. Aggressive pituitary tumours and carcinomas: two sides of the same coin? Eur J Endocrinol. 2018; 178(6): C7-C9, doi: 10.1530/EJE-18-0250, indexed in Pubmed: 29588294.

6. Lee JH, Miele ME, Hicks DJ, et al. KiSS-1, a novel human malignant melanoma metastasis-suppressor gene. J Natl Cancer Inst. 1996; 88(23): 1731-1737, doi: 10.1093/jnci/88.23.1731, indexed in Pubmed: 8944003.

7. West A, Vojta PJ, Welch DR, et al. Chromosome localization and genomic structure of the KiSS-1 metastasis suppressor gene (KISS1). Genomics. 1998; 54(1): 145-148, doi: 10.1006/geno.1998.5566, indexed in Pubmed: 9806840.

8. Kotani M, Detheux M, Vandenbogaerde A, et al. The metastasis suppressor gene KiSS-1 encodes kisspeptins, the natural ligands of the orphan G protein-coupled receptor GPR54. J Biol Chem. 2001; 276(37): 34631-34636, doi: 10.1074/jbc.M104847200, indexed in Pubmed: 11457843.

9. Lee DK, Nguyen T, O'Neill GP, et al. Discovery of a receptor related to the galanin receptors. FEBS Lett. 1999; 446(1): 103-107, doi: 10.1016/s0014-5793(99)00009-5, indexed in Pubmed: 10100623.

10. Harihar S, Ray S, Narayanan S, et al. Role of the tumor microenvironment in regulating the anti-metastatic effect of KISS1. Clin Exp Metastasis. 2020; 37(2): 209-223, doi: 10.1007/s10585-020-10030-6, indexed in Pubmed: 32088827.

11. Schmid K, Wang X, Haitel A, et al. KiSS-1 overexpression as an independent prognostic marker in hepatocellular carcinoma: an immunohistochemical study. Virchows Arch. 2007; 450(2): 143-149, doi: 10.1007/s00428-006-0352-9, indexed in Pubmed: 17216189.

12. Marot D, Bieche I, Aumas C, et al. High tumoral levels of Kiss1 and G-protein-coupled receptor 54 expression are correlated with poor prognosis of estrogen receptor-positive breast tumors. Endocr Relat 
Cancer. 2007; 14(3): 691-702, doi: 10.1677/ERC-07-0012, indexed in Pubmed: 17914099.

13. Jarzabek K, Koda M, Kozlowski L, et al. Immunohistochemical study of KiSS1 and KiSS1R expression in human primary breast cancer: Association with breast cancer receptor status, proliferation markers and clinicopathological features. Histol Histopathol. 2015; 30(6): 715-723, doi: 10.14670/HH-30.715, indexed in Pubmed: 25535062.

14. Trevisan CM, Montagna E, de Oliveira R, et al. Kisspeptin/GPR54 System: What Do We Know About Its Role in Human Reproduction? Cell Physiol Biochem. 2018; 49(4): 1259-1276, doi: 10.1159/000493406, indexed in Pubmed: 30205368.

15. Martínez-Fuentes AJ, Molina M, Vázquez-Martínez R, et al. Expression of functional KISS1 and KISS1R system is altered in human pituitary adenomas: evidence for apoptotic action of kisspeptin-10. Eur J Endocrinol. 2011; 164(3): 355-362, doi: 10.1530/EJE-10-0905, indexed in Pubmed: 21169415.

16. Yaron M, Renner U, Gilad S, et al. KISS1 receptor is preferentially expressed in clinically non-functioning pituitary tumors. Pituitary. 2015 ; 18(3): 306-311, doi: 10.1007/s11102-014-0572-y, indexed in Pubmed: 24817066.

17. Kononen J, Bubendorf L, Kallioniemi A, et al. Tissue microarrays for high-throughput molecular profiling of tumor specimens. Nat Med. 1998; 4(7): 844-847, doi: 10.1038/nm0798-844, indexed in Pubmed: 9662379.

18. Kostakis ID, Agrogiannis G, Vaiopoulos AG, et al. A clinicopathological analysis of KISS1 and KISS1R expression in colorectal cancer. APMIS. 2015; 123(7): 629-637, doi: 10.1111/apm.12397, indexed in Pubmed: 26010933.

19. Sun YB, Xu S. Expression of KISS1 and KISS1R (GPR54) may be used as favorable prognostic markers for patients with non-small cell lung cancer. Int J Oncol. 2013; 43(2): 521-530, doi: 10.3892/ijo.2013.1967, indexed in Pubmed: 23716269.

20. Sanchez-Carbayo M, Capodieci P, Cordon-Cardo C. Tumor suppressor role of KiSS-1 in bladder cancer: loss of KiSS-1 expression is associated with bladder cancer progression and clinical outcome. Am J Pathol. 2003; 162(2): 609-617, doi: 10.1016/S0002-9440(10)63854-0, indexed in Pubmed: 12547718.

21. Fratangelo F, Carriero MV, Motti ML. Controversial Role of Kisspeptins/KiSS-1R Signaling System in Tumor Development. Front
Endocrinol (Lausanne). 2018; 9: 192, doi: 10.3389/fendo.2018.00192, indexed in Pubmed: 29760678.

22. Ringel MD, Hardy E, Bernet VJ, et al. Metastin receptor is overexpressed in papillary thyroid cancer and activates MAP kinase in thyroid cancer cells. J Clin Endocrinol Metab. 2002; 87(5): 2399, doi: 10.1210/jcem.87.5.8626, indexed in Pubmed: 11994395 .

23. Savvidis C, Papaoiconomou E, Petraki C, et al. The role of KISS1/KISS1R system in tumor growth and invasion of differentiated thyroid cancer. Anticancer Res. 2015; 35(2): 819-826, indexed in Pubmed: 25667462.

24. Manojlovic-Gacic E, Bollerslev J, Casar-Borota O. Invited Review: Pathology of pituitary neuroendocrine tumours: present status, modern diagnostic approach, controversies and future perspectives from a neuropathological and clinical standpoint. Neuropathol Appl Neurobiol. 2020; 46(2): 89-110, doi: 10.1111/nan.12568, indexed in Pubmed: 31112312.

25. Nash KT, Phadke PA, Navenot JM, et al. Requirement of KISS1 secretion for multiple organ metastasis suppression and maintenance of tumor dormancy. J Natl Cancer Inst. 2007; 99(4): 309-321, doi: 10.1093/jnci/djk053, indexed in Pubmed: 17312308.

26. Richard N, Galmiche G, Corvaisier S, et al. KiSS-1 and GPR54 genes are co-expressed in rat gonadotrophs and differentially regulated in vivo by oestradiol and gonadotrophin-releasing hormone. J Neuroendocrinol. 2008; 20(3): 381-393, doi: 10.1111/j.1365-2826.2008.01653.x, indexed in Pubmed: 18208554 .

27. Hershey JWB, Sonenberg N, Mathews MB. Principles of Translational Control. Cold Spring Harb Perspect Biol. 2019; 11(9), doi: 10.1101/cshperspect.a032607, indexed in Pubmed: 29959195.

28. Torlakovic EE, Cheung CC, D'Arrigo C, et al. From the International Society for Immunohistochemistry and Molecular Morphology (ISIMM) and International Quality Network for Pathology (IQN Path). Evolution of Quality Assurance for Clinical Immunohistochemistry in the Era of Precision Medicine. Part 3: Technical Validation of Immunohistochemistry (IHC) Assays in Clinical IHC Laboratories. Appl Immunohistochem Mol Morphol. 2017; 25(3): 151-159, doi: 10.1097/PAI.0000000000000470, indexed in Pubmed: 28187030.

29. Taylor CR. Milestones in Immunohistochemistry and Molecular Morphology. Appl Immunohistochem Mol Morphol. 2020; 28(2): 83-94, doi: 10.1097/PAI.0000000000000833, indexed in Pubmed: 32044876. 